\title{
MITOS Y ASUNCIONES DE LAS ESCUELAS CHÁRTER: UN ANÁLISIS DE LOS COLEGIOS EN CONCESIÓN DE BOGOTÁ*
}

\author{
Andreu Termes ${ }^{1}$ \\ Antoni Verger ${ }^{1}$ \\ XAVIER BONAL ${ }^{1}$
}

\begin{abstract}
RESUMEN: Los Colegios en Concesión (CEC) son un programa de escuelas chárter que se implementa en Colombia desde el año 1999. A pesar de su modesta expansión (72 escuelas, 107.000 alumnos), los CEC disfrutan de un amplio reconocimiento internacional tanto académico como político (p.ej., por parte del Banco Mundial o el Banco Interamericano de Desarrollo), y son considerados como un modelo paradigmático y en gran medida exitoso de escuelas chárter. Basado en el marco teórico-metodológico de la evaluación realista, y a partir de un trabajo de campo que incluye técnicas tanto cualitativas como cuantitativas, el artículo cuestiona las hipótesis y los resultados del programa de CEC y, a su vez, discute las asunciones generales del modelo chárter.
\end{abstract}

Palabras clave: Chárter. Colegios en Concesión. Bogotá. Evaluación realista.

\section{CHARTER SCHOOLS' MYTHS AND ASSUMPTIONS: ANALYZiNG THE “COLEgios En CONCESIÓN” OF BOgotá}

\begin{abstract}
The "Colegios en Concesión" (CEC) are a charter school program being implemented in Colombia since 1999. Despite its modest expansion (72 schools, 107,000 students), the CEC enjoy international recognition in both academic and policy practice fields (e.g., for the World Bank or the Inter-American Development Bank); in these fields, CECs are generally considered as a paradigmatic and, to a great extent, successful model of charter schools. Based on the theoretical-methodological framework of realistic evaluation, and through a fieldwork that includes both qualitative and quantitative techniques, this article challenges the hypothesis and results of the CEC program and, at the same time, discusses the general assumptions of the charter schools' model.
\end{abstract}

Keywords: Charter. Colegios en Concesión. Bogotá. Realistic evaluation.

\footnotetext{
*Este artículo es resultado de los proyectos Educational Inequalities and Public-Private Partnerships in Colombian Education con el apoyo de PERI-Private Education Research Initiative, EDUPARTNER (Ref. GA-2012-322350, EU Program "PEOPLE”), y EDUMERCAL (CSO2011-22697), además de contar con la colaboración de la Fundación Escuela para el Desarrollo de Bogotá.

${ }^{1}$ Universitat Autónoma de Barcelona (UAB) - Barcelona, Espańa. E-mails: andreu.termes@uab.cat, antoni.verger@uab.cat, xavier.bonal@uab.cat
}

DOI: 10.1590/ES0101-73302017173097 


\title{
LES MYTHES ET LES SUPPOSITIONS DES ÉCOLES CHARTER: unE ANALYSE DES « Colegios EN ConCESSION 》 DE Bogotá
}

\begin{abstract}
RÉSUMÉ: Les "Colegios en Concesión " (CEC) sont un programme charter qui est mis en œuvre en Colombie depuis 1999. En dépit de sa modeste expansion (72 écoles, 70.000 étudiants), les CEC bénéficient d'une large reconnaissance internationale à la fois académique et politique (par exemple par la Banque Mondiale ou la Banque Interaméricaine du Développement), et sont considérés comme un modèle paradigmatique et, en grande parti, brillant des écoles charter. Sur la base du cadre théorique et méthodologique de l'évaluation réaliste, et à partir d'un travail de terrain qui comprend des techniques à la fois qualitatives et quantitatives, l'article questionne les hypothèses et les résultats du programme des CEC et, à son tour, examine les hypothèses générales du modèle des écoles charter.
\end{abstract}

Mots-clés: Écoles charter. Colegios en Concesión. Bogotá. Évaluation réaliste.

\section{Introducción}

$\mathrm{L}$

as escuelas chárter son escuelas gestionadas por entidades privadas, pero están financiadas con fondos públicos y habitualmente son de titularidad pública. Se trata de un modelo escolar bastante extendido en los Estados Unidos, donde ha generado un intenso debate sobre política educativa y acerca del rol del sector privado en educación. Los defensores del modelo chárter, como la National Alliance for Public Charter Schools, consideran que, entre otras virtudes, las escuelas chárter son más eficientes y más transparentes que las escuelas públicas; contratan a profesorado altamente cualificado y motivado; son inclusivas e innovadoras; y obtienen mejores resultados académicos que las escuelas públicas (NAPCS, 2014). En cambio, académicos críticos consideran que el modelo chárter no solamente no cumple con sus promesas de innovación y excelencia, sino que generan discriminación educativa y más desigualdades (BULKLEY \& FISLER; 2003; LUBIENSKI, 2003). Para estos últimos, el modelo chárter se ha construido sobre la base de una serie de mitos y asunciones alrededor de la superioridad del sector privado en la gestión de servicios educativos, aunque esta supuesta superioridad cuenta con poca evidencia empírica.

El objetivo de este artículo es analizar hasta qué punto las expectativas generadas por el modelo de escuelas chárter se cumplen o no en el contexto colombiano y, concretamente, en el caso de los Colegios en Concesión de Bogotá. Los Colegios en Concesión (CEC) constituyen el programa de escuela chárter con mayor tradición y más emblemático de América Latina. A pesar de ello, y a diferencia de la experiencia de las escuelas chárter de EE.UU., todavía contamos con poca literatura académica sobre 
este programa. El objetivo de este artículo es analizar hasta qué punto las supuestas virtudes de este modelo de provisión educativa en el terreno de la eficacia y de la equidad efectivamente se cumplen para el caso de los CEC de Bogotá.

El artículo se estructura del modo siguiente. El primer apartado expone las características generales del modelo de escuelas chárter. El segundo apartado presenta el programa de los CEC y su emergencia en el contexto educativo colombiano; también revisa de forma esquemática los principales resultados de las evaluaciones que se han hecho del programa. En un tercer apartado se presenta la metodología de nuestra investigación, que, como detallaremos, se basa en la aproximación conocida como evaluación realista. El cuarto apartado presenta los principales resultados de la investigación relativos a aspectos organizativos y pedagógicos, a los principales resultados educativos de las escuelas, y a la relación de las escuelas con su entorno. Finalmente, en el último apartado del artículo discutimos los resultados obtenidos y sus implicaciones en materia de política educativa.

\section{Características y difusión de las escuelas chárter}

Los planteamientos favorables a la privatización y, en concreto, a las Alianzas Público-Privadas (APPs), resuenan con fuerza en la agenda educativa global (ROBERTSON \& VERGER, 2012). Junto a las políticas de voucher escolar, las escuelas chárter representan la modalidad de APP más conocida y debatida tanto en países del norte como del sur (PATRINOS et al., 2009). Los inicios de las escuelas chárter se remontan al año 1991, cuando el estado de Minnesota aprobó una ley que permitía este tipo de provisión educativa. Desde entonces, la legislación pro-chárter se extendió a muchos estados norteamericanos, en parte debido al apoyo que recibieron del partido demócrata cuando Bill Clinton ocupaba la Casa Blanca. La ambigüedad retórica de las chárter les ha permitido ser una opción transversal y bipartidista, implementada por estados con gobiernos tanto demócratas como republicanos. Concretamente, las chárter representan una segunda opción de consenso frente a reformas de mercado más drásticas como los vouchers universales (BULKLEY 2004; 2005). El modelo de escuelas chárter se ha expandido también a escala internacional, y Colombia es uno de los países que de forma más persistente en el tiempo ha implementado este modelo (BARRERA-OSORIO et al., 2012).

Las chárter son una modalidad de escuelas híbridas: de propiedad y financiación estatal, pero gestionadas privadamente y exentas de diversas regulaciones estatales (LUBIENSKI, 2003). Según sus defensores, las escuelas chárter permiten combinar alta autonomía escolar con elevada rendición de cuentas (FINNIGAN, 2007). Dicha combinación, sobre todo cuando tiene lugar en entornos competitivos (p.ej., ante la posibilidad de elección de centro, financiación a la demanda, o cuando hay información pública sobre resultados escolares disponible), genera supuestamente una serie de beneficios tanto desde el lado de la oferta 
como del de la demanda. Así, las familias tienen libertad para escoger el centro que más se ajuste a sus preferencias. Asimismo, las escuelas, que compiten por el alumnado, se ven obligadas a dar respuesta a las necesidades y preferencias de las familias y, por extensión, a mejorar e innovar pedagógicamente. Además, esta excelencia académica es accesible a todo tipo de alumnado, ya que las chárter combinan la gratuidad fruto de la financiación pública con la obligación de aceptar todo tipo de alumnado. Brindar excelencia académica al alumnado más vulnerable es la raison d'être de este modelo. Este elemento es clave para su legitimidad política, e incluso ha generado "un argumento basado en el derecho de acceso a una educación de calidad mediante la elección escolar" (LUBIENSKI et al., 2009, p. 603).

Los defensores de las escuelas chárter se adscriben por regla general a los postulados de la public choice theory (PCT), según los cuales el sector público carece de suficientes incentivos competitivos (y está más bien sometido a intereses corporativos y profesionales ajenos a los de los "usuarios"), lo que deriva en una gestión burocrática sobre-regulada, e ineficiente tanto a nivel económico y organizativo, como pedagógico (CHUBB \& MOE, 1990). Además, el sector público (sin incentivos, y frecuentemente centralizado) no promueve la innovación pedagógica, lo que deriva en un estilo educativo homogéneo y monolítico. En contraposición, se concibe el sector privado como ágil y competitivo, que da respuesta a las preferencias de familias y alumnado, y en constante pugna por diferenciarse de otros proveedores a través de la innovación pedagógica (lo que en último término deriva en un aumento de la diversidad de la oferta escolar). En este esquema, se asume que las chárter disfrutan de la superioridad de los modelos organizativos de estilo privado, en distintas dimensiones: autonomía; rendición de cuentas; eficiencia económica; generación de competencia e innovación pedagógica; resultados académicos; y accesibilidad (véase una síntesis de las asunciones al respecto en el Cuadro 1).

Por supuesto, más allá de las asunciones genéricas del modelo chárter, cabe tener en cuenta la 'adopción selectiva' (PECK \& THEODORE, 2010) de dicho modelo en diferentes contextos locales (BULKLEY, 2005; BULKLEY \& FISLER, 2003). No obstante, como veremos a continuación, el modelo de escuela chárter adoptado en Bogotá se basa en una teoría del cambio que, a pesar de sus especificidades, incluye muchas de las asunciones del modelo global sintetizado en el Cuadro 1.

\section{Los Colegios en Concesión y su contexto}

Colombia es uno de los países latinoamericanos que ha experimentado de forma más entusiasta con las APPs en el campo educativo (BARRERA-OSORIO et al., 2012). No obstante, la privatización educativa en Colombia ha avanzado de forma irregular, y sobre todo a través de la adopción de reformas educativas a nivel subnacional. Entre las APPs educativas más importantes destacan los Colegios en Convenio, el ya extinguido sistema de voucher escolar PACES, y los CEC ${ }^{1}$. 
La expansión de proveedores educativos privados y de APPs en Colombia se explica en gran medida por la infrafinanciación histórica del sector educativo público en el país. Así, el gasto del gobierno colombiano en educación como porcentaje del PIB es solo del 4,8\% (cifra inferior a la media regional de América Latina y el Caribe). Como consecuencia de esta infrafinanciación, Colombia presenta una oferta pública insuficiente (p.ej., solo en Bogotá se estima un déficit de más de 31 mil plazas escolares). Además, las tasas de matriculación alcanzan solamente el $63 \%$ en preprimaria, $87 \%$ en primaria, $72 \%$ en secundaria inferior, y $41 \%$ en secundaria superior, cifras inferiores a la media de América Latina y el Caribe (UNESCO-UIS, 2012). En este contexto, la estrategia política para aumentar el acceso educativo ha consistido en aumentar la ratio alumnado/profesorado (MIÑANA, 2006) y, también, las jornadas escolares múltiples. De hecho, los colegios públicos ofrecen jornadas escolares de mañana (7am-12pm), tarde (12-4pm), e incluso noche (2-7pm) (BARRERA-OSORIO et al., 2012). Solo el 0,8\% del alumnado de los colegios públicos disfruta de una jornada escolar completa.

La baja expansión del sector público ha estimulado el incremento del número de escuelas privadas por todo Colombia, pero especialmente en ciudades como Bogotá, donde el sector privado da cobertura al $62 \%$ del alumnado matriculado en preprimaria, al $35 \%$ del alumnado en primaria, al 34\% en secundaria inferior, y al $38 \%$ en secundaria superior. En este contexto de necesidad de expansión educativa $-\mathrm{y}$ de restricciones presupuestarias — emergen los CEC.

\section{Cuadro 1}

Asunciones del modelo de escuela chárter.

1. Las chárter son autónomas a distintos niveles y en varias dimensiones: pedagógica y gerencial.

2. Las chárter facilitan la rendición de cuentas (accountability), ya que combinan una rendición de cuentas de mercado (deben responder ante las familias ya que dependen de su capacidad de atraer demanda) con la rendición de cuentas contractual-legal (deben responder ante la administración, y cumplir con las obligaciones derivadas de su contrato con aquella).

3. Las chárter son eficientes económicamente, resultado de una organización privada y autónoma caracterizada por una gestión de los recursos humanos flexible y con fuertes incentivos económicos. Esto convierte a las chárter en una opción de expansión educativa costo-eficiente para la administración.

4. Las chárter innovan pedagógicamente, lo que contrasta con la ausencia de innovación característica del sector público. La autonomía de las chárter, en un contexto de competencia por el alumnado, permite a estas escuelas dar respuesta a las preferencias de familias y alumnado (Lubienski 2003; Finnigan 2007). Como resultado agregado, las chárter generan mayor diversidad en el espacio educativo local -lo que, en último término permite una mayor capacidad de elección efectiva por parte de las familias.

5. Las chárter son accesibles a todo el alumnado: son gratuitas (gracias a la financiación pública que reciben), y están obligadas a aceptar todo tipo de alumnado independientemente de su condición económica, étnica, o educativa.

6. Las chárter aumentan la competencia entre escuelas tanto públicas como privadas, lo cual mejora los resultados de ambas -ya que expulsa del mercado local a las escuelas con bajo rendimiento. 
Los CEC son escuelas chárter construidas por la Secretaría de Educación de Bogotá (SED) en 1999 - aunque posteriormente expandidas por el resto del país-, que fueron licitadas para ser gestionadas por distintas CMOs (o Charter Management Organizations) hasta $2014^{2}$. Para licitar, los CMOs debían ser entidades sin ánimo de lucro, y contar con experiencia y "excelencia académica" previa corroborada. Las escuelas se construyeron en zonas de contexto económico bajo, y con escasez de plazas escolares. Hoy en día, los CEC tienen una matrícula de unos 40 mil alumnos/as, equivalente al 2,7\% del total de alumnado de Bogotá, aunque en algunas localidades de la capital llegan a representar entre el 5\% y el 9\% (Usme, Santa Fe, Bosa, o San Cristóbal).

El contrato entre la SED de Bogotá y los CEC incluía las obligaciones siguientes:

- La garantía de ciertos estándares relativos a infraestructura, materiales pedagógicos, y servicios (jornada escolar completa, calidad en la alimentación, etc.);

- el logro de resultados académicos (repetición, abandono, o en las pruebas estatales del Instituto Colombiano para el fomento de la Educación Superior -ICFES), superiores a los de las escuelas públicas de su entorno;

- la aplicación de sistemas de selección en el proceso de matriculación de alumnado basados en criterios de proximidad geográfica, situación socio-económica, población vulnerable -como desplazados-, y unificación familiar; y

- la prohibición del lucro.

En el inicio de los procesos de licitación, las altas exigencias de las licitaciones (p.ej., nulos beneficios económicos, requisitos de excelencia académica previa) redujo el número de potenciales ofertantes (aspecto que disminuyó la competencia ex ante), y limitó el perfil de los licitadores, con motivaciones más próximas a la vocación social, al proselitismo religioso, o a la imagen y el prestigio, que al lucro (PÉREZ \& RIVERA, 2010). Paralelamente al proceso de licitación, la SED se comprometía a encargar periódicamente a instituciones independientes las evaluaciones de estos centros. En caso de incumplimiento de las obligaciones, la SED debía cancelar los contratos con los CEC.

A pesar de su reducida importancia cuantitativa, los CEC han capturado la atención de organizaciones internacionales como el Banco Mundial y el $\mathrm{BID}$, así como de académicos independientes, y han sido objeto de múltiples evaluaciones realizadas tanto por estas organizaciones como por la propia administración pública bogotana ${ }^{3}$. A continuación se presenta una síntesis de los principales resultados de estas evaluaciones. 
En primer lugar, según un amplio número de evaluaciones, los CEC gozan de mayor autonomía que las escuelas públicas (BARRERA 2006; 2009; BARRERA-OSORIO et al., 2012; VÉLEZ, 2013), si bien dicha autonomía ha tenido un carácter más organizativo que pedagógico (IDEP, 2010; SARMIENTO, 2008). Las familias participan activamente en la definición pedagógica y en el gobierno de los CEC (SARMIENTO, 2008; SARMIENTO et al., 2005), en cursos y talleres ofrecidos por los CEC (CORPOEDUCACIÓN, 2003; 2004), por lo que generan una fuerte identidad colectiva alrededor de estas instituciones (PENA, 2005a). Dicha autonomía es paralela a una elevada rendición de cuentas (DUARTE \& VILLA 2002; SARMIENTO, 2007).

En segundo lugar, las infraestructura y servicios de los CEC son mejores que los de las escuelas públicas (CORPOEDUCACIÓN, 2003; 2004) en equipamientos como bibliotecas, laboratorios, acceso a TIC (SARMIENTO et al., 2005); atención psicológica y sanitaria (PÉREZ; RIVERA, 2010); becas para acceder a la educación superior (SARMIENTO, 2008); alimentación (BARRERA, 2006; 2009); o jornada escolar completa. Además, existe una correlación positiva entre resultados académicos e infraestructura (SARMIENTO et al., 2005), así como entre resultados académicos y jornada escolar completa (BARRERA, 2006; 2009; IDEP, 2010).

En tercer lugar, la eficiencia económica de los CEC y las condiciones laborales del profesorado, han sido el mayor motivo de controversia. Según algunos estudios, los CEC reciben menos pesos por alumno/a que los públicos (DUARTE \& VILLA, 2002) y, por lo tanto son más eficientes, mientras que según otros estudios las cantidades recibidas por los CEC son similares (BONILLA, 2010) o superiores (SARMIENTO el al. 2005; SARMIENTO, 2008) a las recibidas por las escuelas públicas. Las condiciones laborales del profesorado de los CEC son peores que las de los colegios públicos. Se trata de contratos temporales, con jornada laboral más larga (SARMIENTO et al., 2005; SARMIENTO, 2008) y que están sujetos a prácticas ilegales como la no relación entre remuneración y rango profesional (SED, 2004; 2011). Estas condiciones laborales han generado consecuencias ambivalentes: por un lado, la contratación de profesorado poco formado y sin experiencia (SED, 2004; BARRERA, 2006; 2009), o la alta rotación del profesorado y la consiguiente dificultad de consolidar equipos pedagógicos (IDEP, 2007; BONILLA, 2010); por otro lado, el aumento de la productividad derivada de la presión profesional (SARMIENTO, 2008; BONILLA, 2010; DUARTE \& VILLA, 2002).

En cuarto lugar, la transferencia educativa de los CMOs se ha visto limitada por el efecto contexto (PEN̈A, 2005b; IAM, 2004), particularmente en los CMO más elitistas (IDEP, 2007). A nivel de resultados académicos, los CEC presentan mejores índices de deserción, abandono y repetición (IDEP, 2007; 2010; 2011; BARRERA, 2006; BARRERA-OSORIO et al., 2012), aunque la estadística inferencial apunta a que esta diferencia no es significativa (SARMIENTO et al. 2005; SARMIENTO, 2008). Los CEC obtienen mejor puntuación en las pruebas ICFES (IDEP, 2007; 2010; 
2011), y en otras pruebas estandarizadas, si bien no en todas las asignaturas ni siempre de forma estadísticamente significativa - o con una baja significatividad del 0,10 (cf BONILLA, 2010; BARRERA, 2006; 2009; EDWARDS \& HARTLEY, 2015). Sin embargo, buena parte de estos estudios omiten la variable jornada escolar o el perfil socio-económico del alumnado, como variables de control. La continuidad académica de los CEC se da especialmente en la formación profesional post-secundaria (SARMIENTO, 2008), lo que apunta a las dificultades de este perfil de alumnado para acceder a la universidad (PÉREZ \& RIVERA, 2010).

Finalmente, por lo que se refiere al acceso, los CEC han presentado irregularidades en los procesos de matriculación: existen evidencias puntuales de cream skimming (MINANA, 2006; EDWARDS \& HARTLEY, 2015; SARMIENTO et al., 2005). Además, las familias perciben que las conexiones informales facilitan el acceso a las escuelas (SARMIENTO, 2007).

Dada la abundancia de datos y evidencias sobre el funcionamiento de los CEC, ¿̨uál es el valor añadido de nuestro estudio? En primer lugar, a diferencia de la mayoría de las evaluaciones revisadas, el objetivo de este estudio no es solo evaluar el funcionamiento y resultados de los CEC de forma aislada, sino analizar qué consecuencias ha tenido la implementación de los CEC en su entorno más próximo (en términos de innovación, diversificación o segregación, y en relación a otros centros educativos). En segundo lugar, y como explicamos a continuación, el marco teórico-metodológico de este estudio se basa en la evaluación realista, una metodología que -como desarrollamos a continuación- intenta trascender el positivismo y empirismo habitual en la evaluación de políticas públicas, y tiene en cuenta las variantes contextuales de los programas o políticas educativas que son objeto de la evaluación, así como su adopción selectiva ${ }^{4}$.

\section{Evaluación realista y teoría del cambio de los CEC}

A nivel metodológico, el presente artículo se basa en la evaluación realista, una metodología que concibe los programas y las políticas públicas como ideas y teorías sobre el cambio social, que se articulan en contextos sociales y políticos específicos (PAWSON \& TILLEY, 1997). La evaluación realista se basa un doble proceso analítico y metodológico. En primer lugar, reconstruye la teoría del cambio del programa y, en segundo lugar, convierte las asunciones de la teoría del cambio en hipótesis de trabajo, que deben ser contrastadas en escenarios reales.

La teoría del cambio de un programa incluye los objetivos deseados, los mecanismos asumidos, los resultados esperados y los principios legitimadores del programa en cuestión (BULKLEY, 2005). En esta dimensión juegan un papel clave la identificación de los mecanismos, es decir, los procesos a través los cuales se asume que los programas potencialmente funcionarán y tendrán los 
efectos deseados (PAWSON \& TILLEY, 1997). Por otro lado, a partir de dicha teoría del cambio, se analiza la recontextualización del programa en un contexto específico (no meramente geográfico, sino también histórico, político, institucional, y estructural) y el rol de los distintos agentes en dicho proceso (es decir, sus estrategias de implementación y adaptación selectiva de dichas políticas). En este sentido, la evaluación realista tiene por objetivo identificar y analizar la tríada contexto-mecanismos-resultados (tanto esperados como efectivamente obtenidos). Esto implica que distintos mecanismos serán activos solo bajo determinadas circunstancias y diferentes contextos. Por todo ello, la evaluación realista no propone un 'análisis de impacto' o de resultados en el sentido tradicional (p.ej., evaluar si las escuelas chárter funcionan), sino un planteamiento más complejo: "what works for whom in what circumstances and in what respects and how" (PAWSON \& TILLEY, 1997, p. 2).

Estas consideraciones teóricas implican que, metodológicamente, la evaluación realista tiende a privilegiar aproximaciones cualitativas con las que capturar la reflexividad de los agentes (percepciones, interpretaciones, objetivos, y estrategias) sin por eso excluir análisis cuantitativos (VERGER; BONAL, 2013). De acuerdo con los postulados de la evaluación realista, esta investigación se ha basado en un planteamiento metodológico mixto, tanto cualitativo como cuantitativo. La muestra ha incluido tanto CEC como escuelas públicas de la ciudad de Bogotá. El trabajo de campo final ha incluido tanto fuentes primarias (cuestionarios, entrevistas semi-estructuradas, grupos de discusión) como fuentes secundarias (documentación oficial, resultados académicos de las escuelas), tal como se expone en la Tabla 1.

\section{Tabla 1}

Técnicas de análisis.

\begin{tabular}{l|l}
\hline Técnicas & \multicolumn{1}{c}{ Aplicación concreta de las técnicas } \\
\hline $\begin{array}{l}\text { Revisión de } \\
\text { literatura }\end{array}$ & $\begin{array}{l}\text { Análisis sistemático de literatura académica sobre los CEC, y de las } \\
\text { evaluaciones del programa encargadas por la SED. }\end{array}$ \\
\hline $\begin{array}{l}\text { Análisis } \\
\text { documental }\end{array}$ & $\begin{array}{l}\text { Análisis documental de los procesos de licitación de la SED (1999-2003), } \\
\text { y de los 25 contratos entre SED y cada CEC. }\end{array}$ \\
\hline $\begin{array}{l}\text { Entrevistas } \\
\text { semi-estructuradas }\end{array}$ & $\begin{array}{l}\text { Análisis de contenido de entrevistas semi-estructuradas a 6 informantes } \\
\text { clave. Entrevistas semi-estructuradas a directores/as ( } \mathrm{n}=13), \text { profesorado } \\
(\mathrm{n}=36), y \text { familiares }(\mathrm{n}=31) .\end{array}$ \\
\hline $\begin{array}{l}\text { Grupos de } \\
\text { discusión }\end{array}$ & Análisis de contenido de grupos de discusión con alumnado (n = 11). \\
\hline Cuestionarios & Cuestionarios a directores/as y profesorado (n = 1.086). \\
\hline $\begin{array}{l}\text { Análisis estadístico } \\
\text { inferencial }\end{array}$ & $\begin{array}{l}\text { Análisis de regresión múltiple (con las variables de resultados ICFES en 8 } \\
\text { asignaturas, jornada escolar, y grupo socioeconómico) de los resultados de } \\
145 \text { escuelas (25 de las cuales CEC, y el resto escuelas públicas de la misma } \\
\text { área geográfica). }\end{array}$ \\
\hline
\end{tabular}




\section{La teoría del cambio de los CEC}

A partir del análisis de los documentos legales, de la literatura académica, y de las entrevistas a informantes privilegiados, hemos podido identificar las principales premisas que conforman la teoría del cambio de los CEC. Son las siguientes:

- Los CEC tienen la capacidad de mejorar los resultados académicos del alumnado más pobre (BARRERA, 2006; 2009; PATRINOS et al., 2009) gracias a la transferencia de las exitosas fórmulas pedagógicas de los CMOs a contextos de pobreza; a la autonomía pedagógica y a la existencia de completos mecanismos de evaluación. La accountability de los CEC, especialmente en su dimensión legal-contractual, contribuye a aumentar los resultados académicos ya que ante evaluaciones reiteradamente negativas, los colegios pueden ser expulsados del programa (DUARTE; VILLA, 2002, PEÑA, 2005b);

- Los CEC promueven la innovación pedagógica en el aula (en contraposición al modelo homogéneo de los colegios públicos) y en consecuencia la diversidad del sistema educativo local (lo que incrementa a su vez la libertad efectiva de las familias para escoger entre diferentes opciones pedagógicas). Esto es supuestamente resultado de una mayor autonomía, de la mayor diversidad -tanto pedagógica como religiosa- de los distintos CMOs, y de la competencia entre colegios públicos y privados;

- Los CEC permiten a la administración pública expandir la cobertura educativa en un contexto de déficit de plazas escolar y, además, hacerlo de forma rápida y costo-eficiente (CASTRO et al., 2012; PEÑA, 2005a; 2005b). La autonomía organizativa de las escuelas resulta clave también en este ámbito ya que permite gestionar los recursos humanos de forma flexible e incluyendo incentivos (p.ej., vinculando contratación y despido del profesorado a los resultados obtenidos), aspecto que se supone que tendrá efectos sobre un aumento de la productividad del profesorado (SARMIENTO, 2008; PATRINOS et al., 2009). Asimismo, la autonomía puede permitir reducir el peso excesivo de los sindicatos de profesorado y potenciar un rol más gerencial de las direcciones (BARRERA, 2006; 2009; BARRERA-OSORIO et al., 2012);

- Finalmente, el principio legitimador central del programa es el derecho de la población más vulnerable a una educación de calidad y relevancia; esto es, un principio de acción afirmativa o discriminación positiva (PENAA, 2005b; SARMIENTO et al., 2005; SARMIENTO, 2008) con el que, por extensión, aumentar la equidad del sistema educativo. El cumplimiento de este principio requería que los $\mathrm{CEC}$ ofrecieran una educación excelente y, además, atendieran prioritariamente al alumnado más vulnerable, para lo cual el acceso a los CEC debería ser gratuito, focalizado, y priorizado en alumnado 
particularmente vulnerable. Además, otro principio legitimador - aunque de menor importancia- es ampliar la libertad efectiva de elección de centro, dado que los CEC debían aumentar la variedad de la oferta educativa - sobre todo en zonas más empobrecidas (JOLLY, 2009) —, y garantizar así el derecho constitucional de libre elección de centro.

La teoría del cambio de los CEC se resume de forma gráfica en la Figura 1. Los cuadros representan las políticas públicas introducidas, los círculos los resultado esperados tanto en escuelas - gris claro - como en el sistema educativo local — gris oscuro —, las líneas las relaciones causales asumidas, y los rombos los principios legitimadores.

Siguiendo el esquema metodológico de la evaluación realista, la estrategia empírica y analítica se debe centrar en contrastar las asunciones de la teoría del cambio del programa (convertidas en hipótesis de trabajo o preguntas de investigación) en un escenario real (es decir, en su implementación efectiva). Concretamente, el programa CEC bogotano genera una serie de preguntas por

\section{Figura 1}

Teoría del cambio de los CEC de Bogotá.

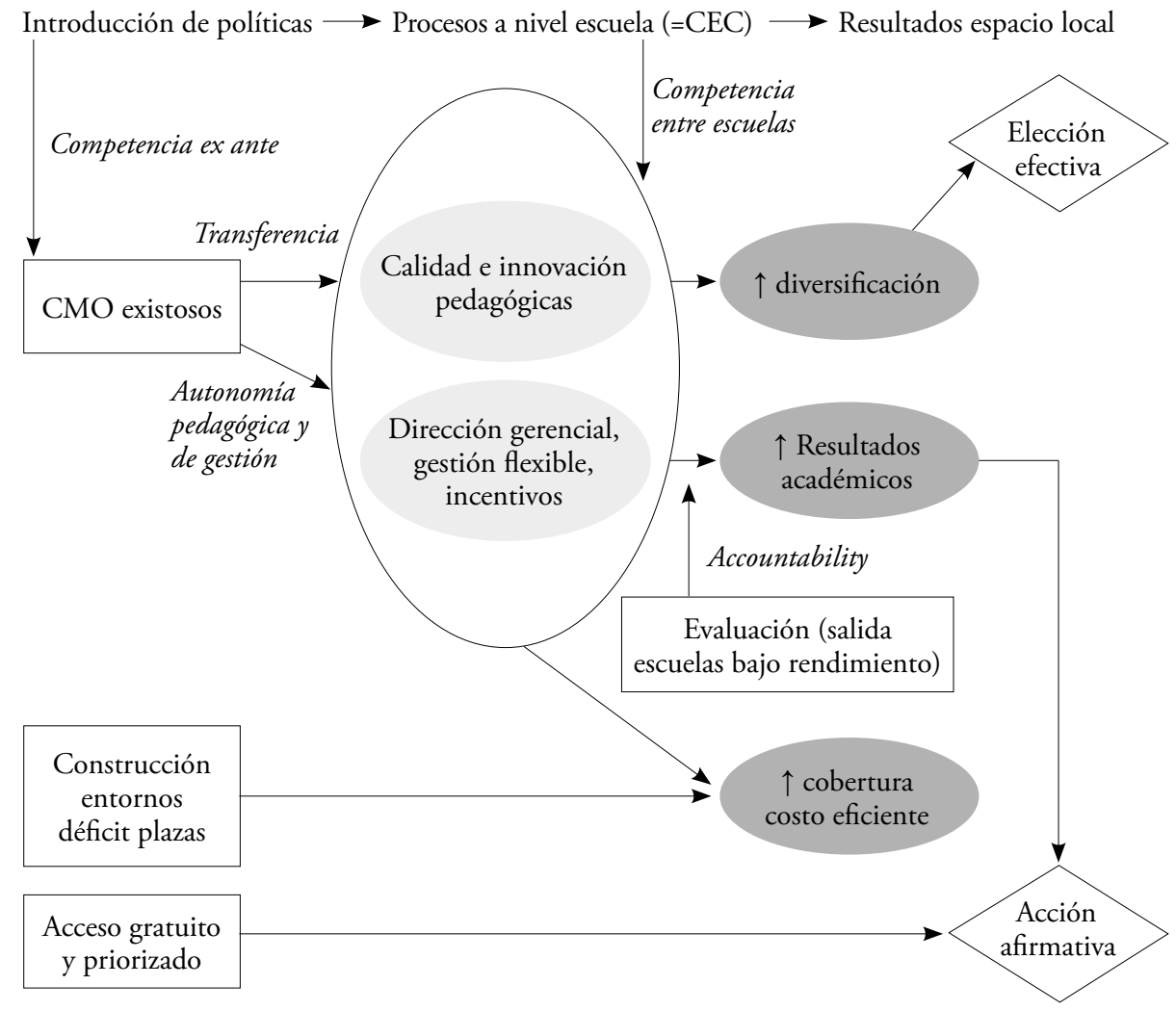


lo que respecta a sus niveles de autonomía, innovación, eficiencia económica, relación con el entorno, rendición de cuentas y rendimiento educativo, que fueron respuestas desde la evaluación realista realizada. A continuación destacamos los principales resultados de nuestro estudio por lo que respecta a dichas cuestiones.

\title{
Resultados: las asunciones de los CEC a prueba
}

¿Hasta qué punto los CEC son más autónomos que las escuelas públicas? El análisis de las entrevistas a directores indica que, en comparación con las escuelas públicas, los CEC son más autónomos que los colegios públicos respecto a la administración pública (la SED). No obstante, los CEC no son realmente autónomos ya que son dependientes de los $\mathrm{CMOs}$ en muchos ámbitos de toma de decisiones. A nivel pedagógico, los CMOs son responsables de elaborar el proyecto de centro de cada CEC afiliado, coordinar el currículo y los materiales pedagógicos, y formar al profesorado. Esto se traslada, p.ej., en una cierta homogenización de proyectos educativos:

\begin{abstract}
"Yo era el asistente de la gerencia de educación de [CMO] y tuve la oportunidad de elaborar la propuesta licitatoria para la SED de estos tres colegios [CEC]" (director, CEC 12).

"Nosotros llegamos acá efectivamente la propuesta de la concesión [durante la licitación] y empezamos a buscar las temáticas, los estándares, de qué se regían en cada área... entonces se hicieron unos grupos de docentes de los cinco colegios, más unos capacitadores y docentes de la [nombre Universidad privada elitista], en conjunto presentamos [el proyecto para todos los CEC]" (Profesora 2, CEC 22).
\end{abstract}

"[Todos los CEC de CMO religioso] llevamos la misma propuesta, los mismos lineamientos... la misma línea” (Profesor 2, CEC 7).

A nivel organizativo, la situación es parecida en determinados aspectos. Los CMOs seleccionan, despiden y definen las condiciones laborales del profesorado, además de estipular los presupuestos escolares (tanto la distribución interna como externa de dichos presupuestos) o de realizar la estimación inicial del costo por alumno/a. A pesar de esta recentralización de los CMOs, los CEC son más autónomos que las escuelas públicas a nivel de gestión, dado el rol más gerencial que adoptan las direcciones de los centros. En general, tanto la autonomía organizativa como pedagógica de los CEC se ha centrado en la capacidad de gestionar políticas de personal, disciplinarias, o pedagógicas, pero no tanto en la capacidad de definir los objetivos o metas educativas.

La autonomía pedagógica debería aumentar la innovación y diversificación pedagógica. Pero ¿hasta qué punto han sido innovadores los CEC? A partir 
de las entrevistas al profesorado, se observa que los CECs, normalmente a través de los CMOs, han introducido cambios en la instrucción en las aulas, pero estos van desde enseńanzas constructivistas y activas (que en ciertos entornos se pueden considerar innovadores) a una educación en valores basada en aspectos como la religiosidad, el emprendimiento o la disciplina en el aula; $y$, de forma generalizada, el teaching to the test (en ocasiones como resultado de la contratación de servicios de simulación de tests ofrecidos por instituciones ajenas a la escuela - e incluso de pago). Esto último contradeciría la idea de una educación adaptada al contexto e innovadora que se pretendía promover con el programa.

¿Son económicamente más eficientes los CEC que las escuelas públicas? Las entrevistas con directores han mostrado como los CEC han adoptado un enfoque de gerencia, lo que ha derivado en una gestión de los recursos humanos más flexible, así como en un esquema de incentivos vinculado con los sistemas de evaluación del profesorado y a mejoras en su situación laboral. Nuestro cuestionario evidencia que las condiciones laborales del profesorado de los CEC son peores que las del profesorado de las escuelas públicas (tal como se muestra a la Tabla 2): jornadas laborales más largas (13 horas semanales más); salarios más bajos; contratos temporales (casi el 99\% del profesorado CEC tiene contratos de 11 meses o menos, en comparación con un sector público caracterizado por contratos indefinidos); y no reconocimiento de los ańos de experiencia. La no correspondencia entre rangos y salarios o la prohibición de sindicalización para el profesorado -común a todos los CEC analizados- es una violación de derechos laborales. A pesar de la relativa

\section{Tabla 2}

Condiciones laborales del profesorado en escuelas públicas y CEC.

\begin{tabular}{l|c|c}
\hline & Escuelas públicas & CEC \\
\hline $\begin{array}{l}\text { Edad, experiencia, } \\
\text { y ańos en la escuela. }\end{array}$ & Edad: 44,4 & Edad: 35,2 \\
Experiencia: 19,3 & Experiencia: 11,4 \\
Años en el colegio: 8,3 & Añ colegio: 4 \\
\hline Rangos más habituales & Especialización: $48,7 \%$ & Profesional: $69 \%$. \\
\hline Estatuto docente & $1979: 51 \%$ & $1979: 36,9 \%$ \\
\hline Jornada laboral en & $2002: 28,7 \%$ & $2002: 63,1 \%$ \\
la escuela y en aula & Escuela: 30,3 & Escuela: 42,9 \\
\hline Tipo contrato & Aula: 23,8 & Aula: 31,2 \\
\hline Salario (en Salarios Mínimos, & Indefinido: $95 \%$ & Indefinido: $1,1 \%$ \\
SM) más habitual & Temporal: $5 \%$ & Temporal: $98,9 \%$ \\
\hline Afiliación sindical & y 4 SM: $83,2 \%$ & 2 o menos SM: $66,5 \%$. \\
\hline
\end{tabular}


satisfacción del profesorado de los CEC, este emigra al sector público en cuanto surge la oportunidad, aspecto que deriva en una elevada movilidad laboral.

Sin embargo, es difícil metodológicamente comparar la eficiencia económica de los CEC y de las escuelas públicas. La SED no tiene la capacidad técnica de estimar el costo por alumno/a en todas los colegios públicos, por lo que solo ofrece el cálculo de las escuelas del sistema $40 * 40$ (es decir, 40 horas semanales, y una ratio de 40 alumnos/profesor), que en realidad solo engloba al 13\% de alumnado del sector público. En esta comparación restringida, el coste por alumno/a de los colegios públicos es de US\$2.091 mientras que el de los CEC es de sólo 1.072 -una diferencia del 51\% (SED 2013). Estas diferencias se explican por dos factores: el salario del profesorado (que representa el $55 \%$ de esta diferencia), y prioridades presupuestarias como transporte o comida (otro $42 \%$ de la diferencia) 5 .

Paralelamente, la gestión de recursos humanos de los CEC también se ha caracterizado por el gran número de actividades de planificación y coordinación pedagógica, muy superior a la de los colegios públicos. Así, tanto la media semanal de planificación pedagógica (encuentros sobre la visión de la escuela; definición del currículo, materiales, o evaluaciones; colaboración entre profesorado; participación en formación continua; entre otras) como también la media semanal de actividades de coordinación de profesorado (observación de clases; solución de problemas conjunta; etc.) de los CEC son significativamente superiores a las de las escuelas públicas (véanse las Tablas 3 y 4). Este número superior de actividades de planificación y coordinación se relaciona tanto con la larga jornada laboral del

Tabla 3

Porcentaje de actividades pedagógicas realizadas mensualmente.

\begin{tabular}{l|c|c}
\hline & $\begin{array}{c}\text { Escuelas } \\
\text { públicas } \%\end{array}$ & $\begin{array}{c}\text { CEC } \\
\%\end{array}$ \\
\hline Reuniones sobre visión y misión del colegio. & 16,2 & 49,2 \\
\hline Desarrollo curricular & 40,8 & 58,7 \\
\hline Discusión sobre material de clase & 34,1 & 48,8 \\
\hline Intercambiar material con colegas & 42,5 & 59,2 \\
\hline Reuniones de equipo de grado & 74,0 & 86,7 \\
\hline Definición sobre evaluación & 43,4 & 64,5 \\
\hline Discutir sobre aprendizaje de determinados alumnos & 65,9 & 81,5 \\
\hline Enseñar conjuntamente con otros colegas & 36,5 & 54,6 \\
\hline Participar en actividades de desarrollo profesional & 22,2 & 57,5 \\
\hline Observar clases de otros colegas & 6,2 & 16,6 \\
\hline Realizar actividades con otros cursos & 36,3 & 52,4 \\
\hline Coordinar tareas con responsables de diferentes áreas & 45,5 & 69,8 \\
\hline
\end{tabular}


profesorado de los CEC como con su situación de inestabilidad laboral (en la que para renovar su contrato deben ajustarse a las altas exigencias de los CEC).

¿Los CEC han transferido y replicado exitosamente las prácticas pedagógicas y de gestión de los CMOs a contextos de pobreza? El contexto socio-económico ha requerido la readaptación de las prácticas pedagógicas habituales de los $\mathrm{CMOs}$ en el marco de los CEC. Dichas prácticas no son tan resilientes como se había asumido en la teoría del cambio del programa CEC. Nuestro análisis cualitativo muestra que tanto profesorado como directores/as perciben que el trasfondo socio-económico del alumnado afecta a los procesos de aprendizaje y a las metodologías que se pueden adoptar:

"La educación no solo es un colegio y unos docentes, hay unos factores asociados, hay un universo socioeconómico, o sea, eso tu no transfieres el modelo del colegio [privado de CMO elitista] que es uno de los colegios más caros de Colombia a Ciudad Bolívar" (Informante clave, responsable de la implementación inicial de los CEC).

Esto se hace especialmente visible en términos de convivencia o violencia, o en las relaciones con las familias (expectativas paternas sobre continuidad académica, colaboración y participación, disponibilidad horaria). Consecuentemente, los CMOs han readaptado sus expectativas y prácticas a estas realidades, y puesto el énfasis en programas de orientación — específicamente readecuadas a familias —, técnicas de re-

Tabla 4

Porcentaje de actividades del director realizadas con alta frecuencia.

\begin{tabular}{l|c|c}
\hline & $\begin{array}{c}\text { Escuelas } \\
\text { públicas }\end{array}$ & CEC \\
\hline Discutir objetivos con profesorado & 57,1 & 92,0 \\
\hline Asegurar la consistencia de prácticas de enseñanza & 65,1 & 94,1 \\
\hline Observar prácticas de enseñanza & 26,5 & 81,2 \\
\hline Sugerir mejoras para mejorar prácticas de enseńanza & 47,3 & 85,0 \\
\hline Reforzar la iniciativa del profesorado & 66,1 & 88,8 \\
\hline Tomar la iniciativa para resolver problemas del profesorado & 46,0 & 70,4 \\
\hline Asegurar oportunidades de formación para el profesorado & 61,3 & 79,6 \\
\hline Felicitar al profesorado que cumple objetivos & 62,8 & 79,7 \\
\hline Apoyar profesores innovadores & 67,0 & 82,3 \\
\hline Trabajar conjuntamente con el profesorado & 72,8 & 95,4 \\
\hline Animar al trabajo en equipo & 78,3 & 94,7 \\
\hline Definir los objetivos del profesorado & 67,3 & 88,3 \\
\hline Promover un buen clima & 82,1 & 94,1 \\
\hline Trabajar juntos para conseguir calidad educativa & 79,3 & 91,9 \\
\hline
\end{tabular}


solución de conflicto, o en una disciplina más estricta en el aula y en el centro. El efecto contexto es más evidente en casos en los que un CMO gestiona CECs en diferentes entornos socio-económicos y, también, en CMOs con tradición de gestión de escuelas en contextos elitistas. Así lo expresan algunos informantes clave:

\begin{abstract}
"Para [CMO elitista] ... manejar grupos de 18 muchachitos ... unos nińitos que llegaban leídos, a llegar a enfrentarse a cursos de $35,40 \ldots$ nos ha llevado hasta a nosotros a cambiar currículos aquí ... esto no es tan fácil tampoco, esto no es que esté resuelto" (informante clave SED).

"Yo creo que en el imaginario de las personas que formularon el proyecto desde el comienzo no cayeron muy en cuenta de la realidad socio económica de estas poblaciones, ellos [CMO elitista en que participa el CEC] hicieron un proyecto educativo muy académico que tiene sentido y vale para nuestra sociedad pero ... eso es muy difícil y sobre todo en esos contextos, igual acá, el contexto aquí socioeconómico es muy pesado, si uno de alguna forma no maneja esos ambientes es muy difícil hacer [un proyecto educativo academicista]" (director CEC 20).
\end{abstract}

\title{
¿Han estado efectivamente controlados los CEC por la administración pública?
} El control institucional del programa CEC ha sido discontinuo y limitado. Por un lado, las evaluaciones externas han sido encargadas a instituciones dependientes de la SED (como el Instituto para la Investigación Educativa y el Desarrollo Pedagógico -IDEP), por lo que se ha cuestionado la legitimidad política de dichas evaluaciones. Además, las evaluaciones han sido metodológicamente discontinuas (con la excepción de IDEP 2010 y 2011). La mayoría de evaluaciones no han tenido consecuencias para los CEC con malas puntuaciones; tan solo la última evaluación ha tenido consecuencias en términos de renovación o discontinuidad de los contratos.

Los controles sobre los procesos internos de los CEC han sido escasos. Aunque los CEC están obligados a seguir los mismos procesos de matriculación de alumnado que las escuelas públicas, las prácticas de selección de alumnado por parte de los CEC son habituales. De hecho, tanto las entrevistas a profesorado, familias, y grupos de discusión a alumnado confirman contundentemente este punto. Así, algunas de las familias entrevistadas y de los grupos de discusión admitieron que durante la matriculación recibieron la visita de una trabajadora social que trabaja para la escuela, que se les exigieron certificados académicos, e incluso que se les aplicaron exámenes ad hoc:

"A mi niño que está en séptimo le hicieron una prueba para poder entrar" (Familia 2, CEC 22).

"No se piden tampoco muchos requisitos, pero si, los más fundamentales, como la conducta, sus resultados académicos...o sea se tiene que ser buen estudiante" (Grupo de discusión, CEC 25). 
“[En la entrevista] yo les digo: 'Mire yo no voy a recibir una persona que sea un parásito que no haga nada contra una persona que trate de luchársela todo el tiempo y trate de ser uno de los mejores, o sea que la tenga clara para qué va a venir a estudiar aquí al colegio ... ¿ ¿usted si va a poder? de verdad que usted me lo promete porque es muy duro"” (Director, CEC 22).

Esta selección de alumnado es fruto de una combinación de factores: la alta demanda para acceder a los CEC, la ambigüedad contractual — que ofrece cierto margen de interpretación por parte de los CMOs sobre los procedimientos de acceso - , y la ausencia de control efectivo por parte de la SED. Así, aunque los CEC cumplen los mínimos requeridos por la SED (proximidad geográfica, condición socio-económica), algunos CEC seleccionan al alumnado con mejor perfil académico. No es posible cuantificar detalladamente la dimensión de esta selección. Nuestro trabajo de campo cualitativo permitió identificar las estrategias utilizadas por los colegios para realizar esta selección: entrevistas con los alumnos/as y familias; consulta del expediente académico previo; y exámenes de entrada. Así pues, para muchos directores/as de los CEC, la intervención activa en la composición del alumnado de los colegios es percibido como una activo estratégico para obtener buenos resultados. En este sentido, el targeting del programa ha sido insatisfactorio: los CEC han potenciado la segregación escolar en los espacios educativos locales en los que se han insertado.

¿Han aumentado los CEC la competencia entre escuelas? Tanto el proceso institucional de matriculación (basado en la zonificación) como el desequilibrio entre demanda y oferta (los CEC han sido construidos en localidades con escasez de plazas escolares) han limitado la capacidad de las familias de elegir entre escuelas públicas y CEC y, en consecuencia, ha reducido la competencia efectiva entre escuelas. Sin embargo, aunque los CEC no han competido por el alumnado, sí que han competido por el alumnado de mejor perfil académico. La teoría de cambio de los CEC considera la competencia escolar como un mecanismo importante para la mejora escolar y la innovación educativa. Pero la competencia necesita múltiples pre-requisitos y condiciones para producirse de forma efectiva. Entre estos pre-requisitos destacan un cierto equilibrio entre demanda y oferta educativa, el cual no se da en absoluto en el caso bogotano debido a un déficit estructural de plazas ${ }^{6}$.

\section{¿Han mejorado los CEC el rendimiento académico del alumnado más po-} bre? Los resultados académicos de los CEC son irregulares. En relación con indicadores internos, los CEC tienen mayor porcentaje de alumnado que aprueba curso $(+7 \%)$, menor repetición (-3\%), y menor abandono (-4\%) que los colegios públicos. Sin embargo, se trata de proxies indirectos, y pueden ser solo indicativos de un tipo de cultura escolar y de instrucción y no corresponder necesariamente con resultados de aprendizaje del alumnado reales.

Nuestro análisis basado en los resultados de las pruebas externas ICFES no muestra diferencias estadísticamente significativas entre CEC y escuelas públicas una vez controladas la jornada escolar y el estatus socio-económico del alumnado 
(SES). El análisis de regresión múltiple evidencia que tanto la jornada escolar como el SES del alumnado son variables más influyentes que el tipo de escuela (CEC, pública) (ver Tabla 5 del anexo). Este análisis cuestiona el supuesto de la superioridad académica per se del sector privado. Por lo tanto, y en línea con lo que demuestran estudios realizados con bases de datos internacionales (véase OECD, 2013, p. 54-57),

\section{Tabla 5}

Regresión linear múltiple con resultados ICFES 2013 (modelo 1 con una variable independiente; modelo 2 con tres variables independientes). Coeficiente B y errores estándar en paréntesis.

\begin{tabular}{|c|c|c|c|c|c|c|c|c|}
\hline & \multicolumn{2}{|c|}{ Matemáticas } & \multicolumn{2}{|c|}{ Química } & \multicolumn{2}{|c|}{ Física } & \multicolumn{2}{|c|}{ Biología } \\
\hline & M1 & M2 & M1 & M2 & M1 & M2 & M1 & M2 \\
\hline Constante & $150,63^{*}$ & $\begin{array}{l}47,905^{*} \\
(1,121)\end{array}$ & $184,28^{*}$ & $\begin{array}{c}48,370^{*} \\
(0,934)\end{array}$ & $192,25^{*}$ & $\begin{array}{l}47,251^{*} \\
(0,876)\end{array}$ & $206,78^{*}$ & $\begin{array}{l}47,462^{*} \\
(0,837)\end{array}$ \\
\hline $\begin{array}{l}\text { Tipo } \\
\text { escuela }\end{array}$ & $1,986^{*}$ & $\begin{array}{l}-2,516^{*} \\
(1,190)\end{array}$ & $3,592^{*}$ & $\begin{array}{l}-0,407 \\
(0,992)\end{array}$ & $4,997^{*}$ & $\begin{array}{c}0,753 \\
(0,931)\end{array}$ & $2,650^{*}$ & $\begin{array}{l}-0,741 \\
(0,889)\end{array}$ \\
\hline $\begin{array}{l}\text { Jornada } \\
\text { escolar }\end{array}$ & - & $\begin{array}{l}3,995^{*} \\
(1,072)\end{array}$ & - & $\begin{array}{l}2,574^{*} \\
(0,894)\end{array}$ & - & $\begin{array}{l}2,126^{*} \\
(0,838)\end{array}$ & - & $\begin{array}{l}2,165^{*} \\
(0,801)\end{array}$ \\
\hline SES 1 & - & $\begin{array}{l}-5,404^{*} \\
(1,202)\end{array}$ & - & $\begin{array}{l}-4,925^{*} \\
(1,002)\end{array}$ & - & $\begin{array}{l}-4,556^{*} \\
(0,940)\end{array}$ & - & $\begin{array}{l}-4,209^{*} \\
(0,898)\end{array}$ \\
\hline SES 2 & - & $\begin{array}{l}-2,447^{*} \\
(1,140)\end{array}$ & - & $\begin{array}{l}-2,823^{*} \\
(0,951)\end{array}$ & - & $\begin{array}{l}-3,331^{*} \\
(0,892)\end{array}$ & - & $\begin{array}{l}-2,363^{*} \\
(0,852)\end{array}$ \\
\hline \multirow[t]{3}{*}{$\begin{array}{l}\text { Adjustado } \\
\mathrm{R}^{2}\end{array}$} & 0,022 & 0,340 & 0,084 & 0,361 & 0,157 & 0,399 & 0,045 & 0,312 \\
\hline & \multicolumn{2}{|c|}{ Filosofía } & \multicolumn{2}{|c|}{ Inglés } & \multicolumn{2}{|c|}{ Lengua } & \multicolumn{2}{|c|}{ Saber Social } \\
\hline & M1 & M2 & M1 & M2 & M1 & M2 & M1 & M2 \\
\hline Constante & $175,83^{*}$ & $\begin{array}{l}43,072^{*} \\
(0,860)\end{array}$ & $145,229^{*}$ & $\begin{array}{l}47,730^{*} \\
(1,115) \\
\end{array}$ & $231,07^{*}$ & $\begin{array}{l}49,762^{*} \\
(0,754)\end{array}$ & $183,78^{*}$ & $\begin{array}{l}47,837^{*} \\
(0,918)\end{array}$ \\
\hline $\begin{array}{l}\text { Tipo } \\
\text { escuela }\end{array}$ & 1,762 & $\begin{array}{l}-1,544 \\
(0,913)\end{array}$ & $3,229^{*}$ & $\begin{array}{l}-0,154 \\
(1,184)\end{array}$ & $5,312^{*}$ & $\begin{array}{c}0,871 \\
(0,800)\end{array}$ & $4,393^{*}$ & $\begin{array}{c}0,376 \\
(0,975)\end{array}$ \\
\hline $\begin{array}{l}\text { Jornada } \\
\text { escolar }\end{array}$ & - & $\begin{array}{l}2,534^{*} \\
(0,822)\end{array}$ & - & $\begin{array}{l}2,614^{*} \\
(1,066)\end{array}$ & - & $\begin{array}{l}1,739^{*} \\
(0,721)\end{array}$ & - & $\begin{array}{l}2,241^{*} \\
(0,879)\end{array}$ \\
\hline SES 1 & - & $\begin{array}{l}-4,669^{*} \\
(0,922)\end{array}$ & - & $\begin{array}{l}-6,608^{*} \\
(1,196) \\
\end{array}$ & - & $\begin{array}{c}-4,560^{*} \\
(0,809)\end{array}$ & - & $\begin{array}{l}-5,093^{*} \\
(0,985)\end{array}$ \\
\hline SES 2 & - & $\begin{array}{l}-2,305^{*} \\
(0,875)\end{array}$ & - & $\begin{array}{l}-4,445^{*} \\
(1,134) \\
\end{array}$ & - & $\begin{array}{c}-2,979 * \\
(0,767)\end{array}$ & - & $\begin{array}{l}3,254^{*} \\
(0,935)\end{array}$ \\
\hline $\begin{array}{l}\text { Adjustado } \\
\mathrm{R}^{2}\end{array}$ & 0,016 & 0,336 & 0,068 & 0,366 & 0,174 & 0,442 & 0,124 & 0,388 \\
\hline
\end{tabular}

Tipo escuela: públicas $=0 ; C E C=1$; Jornada: no completa $=0$; completa $=1$; SES categoría de referencia $=$ SES 3 . 
la adición/exclusión del SES y la jornada escolar es clave cuando se comparan los resultados académicos de escuelas públicas y de gestión privada, como son los CEC, en el contexto bogotano. En definitiva, la comparación entre resultados académicos de los CEC y las escuelas públicas debe partir del reconocimiento explícito y la inclusión en la comparación de la posición inicial, objetivamente superior, en el caso de los CEC en diversos ámbitos: mejores infraestructuras y material especializado; mejor alimentación y servicios - lo que es fundamental en zonas plagadas con malnutrición crónica (véase ACOSTA, 2012) —, largas jornadas del profesorado que permiten mayor número de actividades de coordinación y planificación pedagógica, o selección del alumnado con mejor perfil académico.

\section{Conclusiones: los CEC y el modelo chárter}

Para concluir, en base a los resultados de nuestro estudio, discutimos algunas de las asunciones en las que se basa el modelo chárter así como sus posibles límites o tensiones.

Una asunción central de la teoría del cambio de las escuelas chárter es que dichas escuelas generan innovaciones pedagógicas en el aula y, como resultado de ello, contribuyen a diversificar la oferta educativa local. Sin embargo, más que innovaciones los CEC han adaptado prácticas pedagógicas pre-existentes ${ }^{7}$, muchas de las cuales con un carácter tradicionalista, lo que les ha permitido diferenciarse de los colegios públicos de su espacio educativo local (véase también IDEP, 2007; SARMIENTO, 2008). En concreto, los CEC han adaptado tanto prácticas pedagógicas de carácter constructivista (enseñanzas activas, aprendizaje cooperativo, trabajo por proyectos), como el back-to-basics (focalizado en los saberes instrumentales básicos), estrategias de teaching to the test, o valores 'tradicionalistas'. Por lo tanto, aunque ciertamente las chárter pueden diversificar los espacios educativos locales, es inexacto asumir que la mera introducción de chárters genera per se innovaciones educativas. Además, las decisiones y cambios pedagógicos de los CEC (sean estos constructivistas como tradicionalistas) no han sido tanto el resultado de la presión externa competitiva (como asumen las teorías de mercados educativos) como de motivaciones intrínsecas. Esto indica que para favorecer la innovación pedagógica no es imprescindible la creación de cuasi-mercados educativos o fomentar la competencia entre escuelas, ya que en ocasiones las motivaciones internas, relacionadas con el ethos de las escuelas o la identidad profesional del personal docente, son incentivos válidos para el cambio educativo (LUBIENSKI, 2003; 2006).

La implantación del modelo chárter está claramente constreñida por el contexto económico, social y cultural. Aunque el mecanismo de transferencia-replicabilidad de experiencias pedagógicas de éxito es central en la teoría del cambio de los CEC, existe una tensión entre, por un lado, la transferencia de 
prácticas pedagógicas estandarizadas de éxito probado y, por otro lado, la readaptación de aquellas estrategias al contexto socio-económico local (cf LAKE et al., 2010; FARRELL et al., 2012). Dado que la educación es una customer-input technology, donde el alumnado forma parte del proceso de aprendizaje, el entorno socio-económico limita el mecanismo de transferencia-replicabilidad y reduce la utilidad potencial de soluciones descontextualizadas o estandarizadas en educación. En consecuencia, no es correcto inferir que la mera introducción de CMOs de probada experiencia en el sistema educativo implique automáticamente la transferencia exitosa de prácticas pedagógicas. Esta premisa es aún más cierta cuando la experiencia previa de las CMOs se ha dado en contextos elitistas, como en el caso de muchos de los CEC de Bogotá.

Otro elemento fundamental de la teoría del cambio de las chárter es la combinación entre autonomía escolar y rendición de cuentas (BULKLEY, 2005; BULKLEY \& FISLER, 2003). Como hemos visto, los CEC son únicamente más autónomos que las escuelas públicas en relación a determinados aspectos. De hecho, los CEC han compaginado procesos de autonomía respecto a la administración con procesos de recentralización en relación con los CMOs. La literatura general sobre escuelas chárter identifica también esta paradoja de la centralización y estandardización en contextos de supuesta autonomía escolar (véase FINNIGAN, 2007; LAKE et al., 2010). La rendición de cuentas vía pruebas estandarizadas acostumbra a desencadenar múltiples comportamientos oportunistas entre las escuelas chárter, incluyendo el uso de pedagogías y procesos educativos adaptados a las pruebas estandarizadas, la imposición de sanciones disciplinarias en fechas cercanas a las pruebas, o la reducción y focalización curricular. En todo caso, la intensificación de estos comportamientos oportunistas es una respuesta tan indeseada como esperable en un contexto de fuerte presión evaluadora.

Las escuelas chárter deberían ser accesibles a toda la población en edad escolar. Sin embargo, en contextos de evaluaciones e incentivos competitivos, otro comportamiento que, desde el punto de vista de la equidad, resulta indeseado en las escuelas chárter es que tienden a seleccionar al alumnado que se adscribe mejor a su modelo educativo, y a excluir al alumnado con problemas de aprendizaje. La constatación de que los CEC -como tantas otras chárter (véase BULKLEY \& FISLER, 2003)- seleccionan al alumnado, pone de relieve la necesidad de discutir qué medidas adoptar (p.ej., mecanismos de control administrativo territoriales) para garantizar realmente (de facto, no solo de iure) un acceso equitativo de las familias a todas las instituciones financiadas públicamente.

El empeoramiento de las condiciones laborales del profesorado es una constante en la literatura académica sobre escuelas chárter. Diversos estudios constatan que el profesorado de las chárter trabaja más horas, tiene menos seguridad laboral, y peor salario, o que tiene dificultades en sindicarse, factores 
que derivan en mayor movilidad laboral. El empeoramiento de las condiciones laborales del profesorado no es una situación anómala de los CEC de Bogotá, sino una práctica sistemática de las escuelas chárter y la vía más rápida con la que cuentan para mejorar su eficiencia económica. Ahora bien, tanto el profesorado de los CEC como de otras chárter combina, paradójicamente, una gran insatisfacción laboral (que los lleva a emigrar al sector público tan pronto como es posible) con una alta satisfacción profesional - resultado de compartir proyectos y filosofías tanto pedagógicas como religiosas con otros colegas y el centro educativo en general.

En definitiva, por lo que se desprende de nuestro trabajo, más allá de cuestiones relacionadas con el diseño específico del programa de los CEC y de su implementación concreta en el contexto bogotano, la teoría del cambio general del programa de escuelas chárter adolece de problemas de consistencia más profundos. A la luz de los datos presentados, los CEC son un claro reflejo de los límites que los gobiernos afrontan cuando intentan promover la innovación y la excelencia educativas en contextos de pobreza a través del sector privado, sin atender suficientemente los problemas estructurales de sus sistemas educativos.

\section{Notas}

1. Por un lado, los Colegios en Convenio, están situados en zonas con escasez de plazas escolares. Reciben un voucher anual renovable y atienden población de bajo estatus socio-económico; además, se caracterizan por disponer de infraestructura precaria, instalaciones poco adecuadas, y profesorado escasamente cualificado. En 2013 el 9\% del alumnado de Bogotá asistía a estas escuelas. Por otro lado, el Programa de Ampliación de Cobertura de la Educación Secundaria (PACES), un voucher vigente entre 1991-1997, que alcanzó una cobertura de unos 125 mil alumnos; el voucher era distribuido a partir de un sistema de lotería, y cubría aproximadamente la mitad de los costes de la matrícula.

2. Recientemente, el contrato ha sido renovado a 18 de los 25 CEC.

3. P.ej., del Banco Mundial (PATRINOS et al., 2009; BARRERA, 2006), el Banco Interamericano de Desarrollo (DUARTE \& VILLA, 2002; VÉLEZ, 2013), o la UNESCO (VALENCIA \& CARRIZOSA, 2013).

4. Las políticas educativas globales raramente se diseminan de forma homogénea y completa, sino que lo hacen como discursos selectivos, ideas incipientes, y modelos sintetizados (PECK \& THEODORE, 2010).

5. Además, los CEC disfrutan de beneficios obtenidos de fuertes externas (CMOs, instituciones afines) como becas, apoyo técnico, cursos de formación, que no aparecen necesariamente en los costes anuales de los CEC. En paralelo, la selección de alumnado por parte de los CEC a la que nos referimos más adelante facilita la instrucción (es decir, alumnado más “barato” de educar). 
6. En muchas localidades en que los CEC se construyeron existía un importante déficit de plazas escolares, particularmente en las localidades de Suba (déficit de 10.766 plazas), Kennedy (8.264), Bosa (7.193), Engativá (2.911), Ciudad Bolívar (1.992) o Usaquén (1.815).

7. Analíticamente, el cambio pedagógico puede categorizarse como: innovación, adaptación, y transferencia -donde, además, la innovación es más costosa que la adaptación o la transferencia (LUBIENSKI, 2003).

\section{Referencias}

ACOSTA, K. La desnutrición en los primeros años de vida: Un análisis regional para Colombia. In: Documentos del Banco de la República de Colombia-Economía regional. Cartagena: Banco de la República, 2012. p. 1-43.

BARRERA, F. The Concession Schools of Bogotá, Colombia. In: CHAKRABARTI, R.; PETERSON, P.E. School Choice International: Exploring Public-Private Partnerships. Cambridge: MIT Press, 2009. p. 193-218.

The Impact of Private Provision of Public Education: Empirical Evidence from Bogotås Concession Schools. World Bank Policy Research Working Paper, v. 4121, p. 1-30, 2006.

BARRERA-OSORIO, F.; MALDONADO, D.; RODRÍGUEZ, C. Calidad de la educación básica y media en Colombia: diagnóstico y propuestas. Bogotá: Universidad de los Andes, 2012.

BONILLA, J.D. Contracting out public schools for academic achievement: Evidence from Colombia. Job Market Papers, p. 1-52, 2010.

BULKLEY, K. Reinventing an idea: The political construction of charter schools. The Journal of Educational Foundations, v. 18, n. 1, p. 5-31, 2004.

. Understanding the charter school concept in legislation: the cases of Arizona, Michigan and Georgia, International Journal of Qualitative Studies in Education, v. 18, n. 4, p. 527-554, 2005.

BULKLEY, K.; FISLER, J. A decade of charter schools: from theory to practice. Educational Policy, v. 17, n. 3, p. 317-342, 2003.

CASTRO, J.O.; MARTÍNEZ, A.P.; GALLEGO, A.A. Historia institucional Secretaría de Educación Distrital. Bogotá: SED, 2012.

CHUBB, J.; MOE, T. Politics, Markets, and America's Schools. Washington, D.C.: Brookings Institution, 1990.

CORPOEDUCACIÓN. Informe de gestión: 2003. Bogotá: Corpoeducación, 2003. Informe de gestión: 2004. Bogotá: Corpoeducación, 2004.

DUARTE, J.; VILLA, L. Los colegios en concesión de Bogotá, Colombia. Bogotá: Banco Interamericano de Desarrollo, 2002. 
EDWARDS, B.; HARTLEY, H. Accountability and Competition for Charter Schools? Theory versus Reality in Charter Schools in Bogotá, Colombia. New York: Privatisation in Education Research Initiative Working Papers, 2015.

FARRELL, C.; WOHLSTETTER, P.; SMITH, J. Charter Management organizations an emerging approach to scaling up what works. Educational Policy, v. 26, n. 4, p. 499-532, 2012.

FINNIGAN, K.S. Charter school autonomy the mismatch between theory and practice. Educational Policy, v. 21, n. 3, p. 503-526, 2007.

INSTITUTO ALBERTO MERANI (IAM). Una propuesta para cualificar la convivencia de los jóvenes bogotanos escolarizados. Bogotá: Ministerio de Educación Nacional, 2004.

INSTITUTO PARA LA INVESTIGACIÓN EDUCATIVA Y EL DESARROLLO PEDAGÓGICO (IDEP). Informe final de la evaluación a colegios en concesión. Bogotá: SED, 2011.

. Resultados de la III fase de evaluación a colegios en concesión. Bogotá: SED, 2010.

Seguimiento y evaluación al modelo de gestión educativa por concesión. Bogotá: SED, 2007.

JOLLY, J. Descentralización, políticas públicas, gobernancia y territorio. Papel Politico, v. 14, n. 1, p. 107-141, 2009.

LAKE, R.; DUSSEAULT, B.; BOWEN, M.; DEMERITT, A.; HILL, P. The National Study of Charter Management Organization (CMO) Effectiveness. Washington, D.C.: Center on Reinventing Public Education, 2010.

LUBIENSKI, C. Incentives for School Diversification. Journal of School Choice, v. 1, n. 2, p. 1-31, 2006.

. Innovation in Education Markets. American Educational Research Journal, v. 40, n. 2, p. 395-443, 2003.

LUBIENSKI, C.; GULOSINO, C.; WEITZEL, P. School choice and competitive incentives: Mapping the distribution of educational opportunities across local education markets. American Journal of Education, v. 115, n. 4, p. 601-647, 2009.

MIÑANA, C. Estado, mercado y comunidad: reformas educativas y redefinición de lo público en Colombia, 1994-2005. Tesi (Doctorado), Barcelona, Universidad de Barcelona, 2006.

NATIONAL ALLIANCE FOR PUBLIC CHARTER SCHOOLS (NAPCS). Separating Fact\& Fiction: what you need to know about charter schools. Washington, D.C.: National Alliance for Public Charter Schools, 2014.

ORGANISATION FOR ECONOMICCO-OPERATION AND DEVELOPMENT (OECD). PISA 2012 Results: What Makes Schools Successful? Resources, Policies and Practices. PISA: OECD Publishing, 2013. v. 4.

PATRINOS, H.A.; GUÁQUETA, J.; OSORIO, F.B. The Role and Impact of Public-Private Partnerships in Education. Washington, D.C.: World Bank, 2009.

PAWSON, R.; TILLEY, N. Realistic Evaluation. London: SAGE Publications, 1997.

PECK, J.; THEODORE, N. Mobilizing policy: Models, methods, and mutations. Geoforum, v. 41, n. 2, p. 169-174, 2010. 
PEÑA, M. Aprendizajes sobre la gestión educativa. Bogotá: F. Empresarios por la Educación, 2005a.

. Discriminación positiva en las políticas educativas de Bogotá. In: FONDO DE LAS NACIONES UNIDAS PARA LA INFANCIA (UNICEF). Politicas educativas y equidad. Santiago de Chile: Huidobro, 2005b. p. 155-162.

PÉREZ, N.; RIVERA, H.A. Concesión Sabio Caldas: Gimnasio moderno. Bogotá: Universidad del Rosario, 2010.

ROBERTSON, S.L.; VERGER, A. Governing education through public private partnerships. In: VERGER, A.; ROBERTSON, S.; MUNDY, K. (Orgs.). Public private partnerships in education: new actors and modes of governance in a globalizing world. Cheltenham: E. Elgar, 2012. p. 21-42.

SARMIENTO, A. Como avanza el distrito en Educación. Bogotá: SED, 2007.

. Lecciones nacionales de política educativa: Evaluación de becas PACES y colegios en concesión en Bogotá. Foro Semana, Cúcuta, 2008.

SARMIENTO, A.; ALONSO, C.E.; DUNCAN, G.; GARZON, C.A. Evaluación de la gestión de los colegios en concesión en Bogotá 2000-2003. Archivos de Economía, v. 291, p. 1-129, 2005.

SECRETARÍA DE EDUCACIÓN DEL DISTRITO (SED). Informe: 15 años del modelo de concesión. Bogotá: SED, 2013.

Informe de auditoria gubernamental con enfoque integral a la modalidad especial de colegios en convenio (2010-2011). Bogotá: SED, 2011.

Informe final de auditoría gubernamental con enfoque integral modalidad especial. Bogotá: SED, 2004.

UNITED NATIONS EDUCATIONAL, SCIENTIFIC AND CULTURAL ORGANIZATION (UNESCO-UIS). UNESCO Institute for Statistics (UIS) database country profile - Colombia. 2012. Disponível em: <http://uis.unesco.org/>. Acesso em: 27 abr. 2017.

VALENCIA, C.; CARRIZOSA, J. Formación para la paz, la convivencia y la ciudadanía: un análisis de la realidad en seis colegios en concesión de Bogotá. In: ASSY, B. (Dir.). Cátedra UNESCO y Cátedra infancia: Derechos humanos y políticas públicas. Bogotá: Universidad externado de Colombia, 2013. p. 263-308.

VÉLEZ, C. Los Colegios en Concesión en Colombia. PPP Américas. Cartagena, 2013.

VERGER, A.; BONAL, X. La emergencia de las alianzas público-privado en la agenda educativa global. Revista de Currículum y Formación de Profesorado, v. 16, n. 3, p. 11-29, 2013.

Recibido en 05 de diciembre de 2016.

Aprobado en 06 de abril de 2017. 